$\begin{array}{cl}\begin{array}{c}\text { Revue } \\ \text { de Ihistoire } \\ \text { des religions }\end{array} & \text { Revue de l'histoire des religions } \\ & \begin{array}{l}\text { L } 2009 \\ \text { La culture gallicane. Références et modèles (droit, } \\ \text { ecclésiologie, histoire) }\end{array}\end{array}$

\title{
Un gallican sous la Ligue : Guillaume Du Vair
} (1556-1621)

A Gallican in the League: Guillaume Du Vair

\section{Alexandre Tarrête}

\section{OpenEdition}

\section{Journals}

Édition électronique

URL : http://journals.openedition.org/rhr/7280

DOI : $10.4000 /$ rhr.7280

ISSN : 2105-2573

Éditeur

Armand Colin

Édition imprimée

Date de publication : 1 juillet 2009

Pagination : 497-516

ISBN : 978-2200-92591-8

ISSN : 0035-1423

Référence électronique

Alexandre Tarrête, «Un gallican sous la Ligue : Guillaume Du Vair (1556-1621) », Revue de l'histoire des religions [En ligne], 3 | 2009, mis en ligne le 01 juillet 2012, consulté le 05 mai 2019. URL : http:// journals.openedition.org/rhr/7280 ; DOI : 10.4000/rhr.7280 


\section{Un gallican sous la Ligue : Guillaume Du Vair (1556-1621)}

Guillaume Du Vair (1556-1621), conseiller-clerc au Parlement de Paris, ami de Pierre Pithou, de Nicolas Le Fèvre et de Jacques-Auguste de Thou, manifesta pendant la Ligue un vif attachement à la cause gallicane. Chargé avec d'autres parlementaires d'examiner la recevabilité des décrets du Concile de Trente, il attira l'attention sur les dangers que certains articles faisaient courir aux libertés de l'Église de France. Pamphlétaire engagé en faveur d'Henri IV, Du Vair affirma contre les Ligueurs la prééminence du politique sur le religieux, et préféra les intérêts du Royaume aux injonctions de Rome. Sans être lui-même un érudit ou un théoricien, il a beaucoup fait pour diffuser les thèmes gallicans développés par ses amis les « Politiques».

\section{A Gallican in the League : Guillaume Du Vair}

Guillaume Du Vair (1556-1621), counsellor in the Parliament of Paris during the Catholic League crisis and friend of Pierre Pithou, Nicolas Le Fèvre and Jacques-Auguste de Thou, was strongly engaged in favour of the Gallican cause. He examined the articles of the council of Trent and rejected those contrary to the freedom of the French Church. Defending the legitimacy of Henry IV against the League, he valued Politics over Religion and preferred the interests of the French Kingdom to the injunctions of Rome. Without being himself a scholar or a theorist, Du Vair consistently helped spread the ideas of his friends, the "Politiques". 
Guillaume Du Vair (1556-1621) fit sa carrière dans la magistrature. Conseiller-clerc au Parlement de Paris à la fin du règne d'Henri III, il fut nommé maître des requêtes par Henri IV en 1594, puis envoyé en Provence en 1596 pour diriger la chambre de justice de Marseille. Il devint premier président du parlement d'Aix en 1599. Appelé aux sceaux par Marie de Médicis, il revint à Paris en 1616. Révoqué pendant quelques mois, il fut rappelé par Louis XIII après l'assassinat de Concini (1617). La même année, il fut nommé évêque de Lisieux, sans renoncer toutefois à ses fonctions de garde des sceaux. Sacré à Paris en 1619, il ne fit qu'une entrée tardive à Lisieux, où il ne résida guère. Il avait poursuivi le bonnet épiscopal depuis le début des années 1600, et avait même obtenu l'évêché de Marseille, qu'il n'avait finalement pas accepté, mais dont il touchait des revenus qu'il légua dans son testament aux pauvres de l'Hôpital' '. Il semble qu'il conçut la dignité épiscopale comme une source de bénéfices plutôt que comme une charge effective. Il est vrai que celle de garde des sceaux l'accapara jusqu'à la fin de ses jours. Peut-être envisageait-il une sainte retraite à Lisieux, lorsque le roi l'aurait, tôt ou tard, libéré des sceaux.

Son œuvre littéraire date pour l'essentiel du temps de la Ligue : il écrit entre 1584 et 1594 des traités de philosophie d'inspiration stoïcienne ${ }^{2}$ et des méditations bibliques en prose ${ }^{3}$. Il est aussi l'auteur de pamphlets politiques où il exprime à partir de 1592 son engagement en faveur d'Henri IV. Outre ses talents d'écrivain, il était connu pour ses dons d'orateur, qu'il manifesta à maintes reprises : d'abord aux États généraux de la Ligue à Paris en 1593 pour défendre la loi salique et faire obstacle aux ambitions espagnoles sur le trône de France, puis au parlement de Provence lors des remontrances d'ouverture, et enfin comme garde des sceaux de Louis XIII, devant les différentes assemblées du royaume.

1. Voir Edmond Jarno, « Du Vair, évêque de Marseille?», Revue d'Histoire Littéraire de la France, 1954, no 2, p. 195-197.

2. Voir Alexandre Tarrête, «Le stoïcisme chrétien de Guillaume Du Vair (1556-1621) », Stoïcisme et christianisme à la Renaissance, Cahiers V. L. Saulnier, n 23, Paris, Éditions Rue d'Ulm, 2006, p. 93-115.

3. Voir Guillaume Du Vair, Premières Euvres de piété, éd. Bruno PeteyGirard, Paris, Champion, 2002. 
Dans sa vaste étude sur L'Âge de l'éloquence, Marc Fumaroli propose de structurer l'histoire littéraire du début du XVII ${ }^{\mathrm{e}}$ siècle en France autour de l'opposition de deux rhétoriques antagonistes : la rhétorique gallicane et la rhétorique jésuite ${ }^{4}$. Il rattache sans hésitation Guillaume Du Vair à la première, même si ses efforts pour adapter la « rhétorique des citations » à l'éloquence de son temps reviennent à proposer une rupture avec l'éloquence savante des magistrats qui l'ont précédé.

L'appartenance de Du Vair au courant des « Politiques », caractérisé par des valeurs comme le loyalisme monarchique, l'irénisme et le patriotisme, a été souvent soulignée 5 . Je tenterai ici de faire le point sur la dimension spécifiquement gallicane de l'engagement politique de Du Vair. Je m'attacherai à comprendre comment il a été influencé par l'esprit qui animait bon nombre de ses amis (comme Pierre Pithou ou Jacques-Auguste de Thou), et comment cet esprit a guidé son action politique concrète, au service du roi Henri IV, dans le Paris ligueur (de 1592 à 1594), puis dans une Provence fraîchement ralliée à la couronne (de 1596 à 1616).

\section{Affinités gallicanes}

Chez les Du Vair, le gallicanisme est une affaire de famille. Le père de Guillaume, Jean Du Vair - avocat au Parlement de Paris, procureur de Catherine de Médicis et d'Henri d'Anjou, maître des requêtes du duc d'Alençon - fut l'un des protagonistes du grand procès mené contre les jésuites à la fin du XVI siècle 6 . Il s'agissait pour les gallicans de remettre en cause l'attribution à la Société de Jésus d'un legs important de Guillaume Duprat,

4. Voir L'Âge de l'éloquence, Genève, Droz, 1980, p. 673 et suiv.; sur Du Vair, voir p. 500 et suiv.

5. Voir Myriam Yardeni, La Conscience nationale en France pendant les guerres de Religion, Louvain, Nauwelaerts, 1971, chap. 12, p. 297-316, et Alexandre Tarrête, " Guillaume Du Vair et l'héritage de Michel de L'Hospital », De Michel de L'Hospital à l'Édit de Nantes. Politique et religion face aux Eglises, dir. Thierry Wanegffelen, Clermont-Ferrand, Presses Universitaires Blaise Pascal, 2002, p. 575-590.

6. Voir René Radouant, Guillaume Du Vair. L'Homme et l'Orateur jusqu'à la fin des troubles de la Ligue (1556-1596), Paris, 1907, Genève, Slatkine reprints, 1970 , p. 8-14. Le procès connaît de multiples rebondissements et le verdict est longtemps ajourné. 
évêque de Clermont, mort en 1560, ainsi que de contester aux jésuites le droit d'enseigner, voire celui de résider en France. L'avocat Jean Du Vair se trouve alors aux côtés d'Estienne Pasquier, d'Augustin de Thou (président au Parlement) et de Charles Du Moulin (jurisconsulte) pour attaquer les jésuites, dont on dénonce l'inféodation à l'Espagne et à Rome. Mais ces derniers sont défendus par Pierre Versoris, et ils obtiennent pour finir leur part d'héritage; ils fondent alors plusieurs collèges, dont celui de Clermont à Paris.

L'engagement de Jean Du Vair dans le camp des ennemis les plus résolus de la Compagnie de Jésus n'a pas été démenti par les options politiques de son fils. Celui-ci compte lui-même des amis dont l'engagement gallican est notoire : c'est le cas en particulier de Pierre Pithou et de Jacques-Auguste de Thou? ${ }^{7}$. Pierre Pithou, comme Du Vair, reste à Paris pendant la Ligue, et il y publie en 1594 les Libertés de l'Eglise gallicane, chez Mamert Patisson. On conserve une lettre de Du Vair à Pithou qui témoigne de leurs relations amicales ${ }^{8}$. Quant à Jacques-Auguste de Thou, Du Vair le connaît depuis le début des années 1580 au moins ${ }^{9}$. Il entretient avec lui une correspondance suivie et, plus tard, lorsqu'il s'est établi à Aix-en-Provence, De Thou lui envoie régulièrement les volumes de l'Historia sui temporis à mesure qu'ils paraissent (à partir de 1604). À travers ces deux amis, Du Vair est exposé aux thèses gallicanes. Comment ont-elles influencé son action politique?

7. Sur ces auteurs voir en particulier: Les Pithou: les lettres et la paix du royaume, éd. Marie-Madeleine Fragonard et Pierre Leroy, Paris, Champion, 2003; Jacques-Auguste de Thou. Écriture et condition robine, dir. Ingrid De Smet, Paris, Presses Universitaires Paris-Sorbonne, 2007.

8. Voir Du Vair, lettre à Pierre Pithou (s.d., probablement au milieu des années 1590), dans Charles-Alexandre Sapey, Études biographiques pour servir à l'histoire de l'ancienne magistrature française, Paris, 1858, Slatkine reprints, 1971, p. 427-428. Cette lettre mentionne entre autres Jacques-Auguste de Thou et Nicolas Le Fèvre, deux amis communs. Voir aussi la lettre à de Thou sur la mort de leur ami commun Pierre Pithou (novembre 1596), Lettres inédites de G. Du Vair, éd. Philippe Tamizey de Larroque, Paris, Aubry, 1873, p. 45.

9. Voir Alexandre Tarrête, «Le stoïcisme dans la pensée de Jacques-Auguste de Thou ", Jacques-Auguste de Thou. Écriture et condition robine, op. cit., p. 107125. 


\section{L'EXAMEN DES DÉCRETS DU CONCILE DE TRENTE par les États de la Ligue (1593)}

La première occasion où l'on vit Guillaume Du Vair se signaler par son zèle en faveur des libertés gallicanes remonte au temps où il se trouve à Paris, alors aux mains des Ligueurs. Député du TiersÉtat de Paris aux États généraux convoqués par Mayenne en 1593, Du Vair participe à une commission qui doit examiner les décrets du Concile de Trente pour voir s'ils peuvent être reçus en France, comme le légat du pape en a réitéré officiellement la demande. Cette commission est dirigée par deux hommes fort différents : le président Le Maître et l'avocat du roi Louis d'Orléans. Ce dernier, pourtant Ligueur, se fait lui aussi un ardent défenseur des libertés de l'Église gallicane. La recevabilité des décrets du concile, pour lui comme pour les «Politiques », dépend de leur compatibilité avec les traditions du royaume de France. Les procès verbaux des États décrivent ainsi la mission confiée à ces experts :

«La chambre avoit deputé des commissaires pour voir [ledict concile] et verifier si en iceluy il y avoit point quelques articles qui fussent contre les droits de la couronne, libertez, franchises et immunitez de l'eglise gallicane $[\ldots]$ entre lesquels commissaires estoient messieurs le president Le Maistre, et d'Orleans, advocat du roy au Parlement, qui s'estoient chargez de le lire et adviser si ès articles d'iceluy il y avoit aucune chose où l'estat et l'eglise gallicane eussent interes ${ }^{10}$. »

Le libellé de la mission confiée aux parlementaires associe étroitement la défense de l'État royal et celle des prérogatives de l'Église gallicane.

Selon les sources, la présence de Du Vair parmi les membres de cette commission est ou non attestée. Dans ce premier compte rendu, son nom ne figure pas $^{11}$, mais le rédacteur s'en est visiblement tenu aux noms des deux chefs d'équipe. Cependant René Radouant souligne que d'autres textes mentionnent Du Vair ${ }^{12}$. C'est le cas d'un autre compte rendu des États, dû à Odet Souret ${ }^{13}$. De même,

10. Procès-verbaux des Estats généraux de 1593, éd. Auguste Bernard, Paris, 1842 , p. 324.

11. Ni p. 80 , ni p. $324-325$.

12. Voir René Radouant, Guillaume Du Vair, op. cit., p. 316.

13. Voir les Procès-verbaux des Estats généraux de 1593, op. cit., p. 653; Du Vair est cité avec les hommes qui ont secondé le président Le Maître (Bernard, Du Laurens, Le Barbier). 
un fragment retrouvé du Journal de Pierre de L'Estoile associe le nom de Du Vair et celui de Le Maître ${ }^{14}$. Chez de Thou, la présence de Du Vair est moins claire que ne le dit Radouant, car elle varie selon les éditions. Du Vair n'est pas cité dans la première édition posthume (1620) :

«Negotium datum Joanni Magistro quem curiae praesidem Meduanius creaverat et quibusdam aliis viris probis et a factione alienis, juris nostris peritissimis, qui concilium inspicerent $[\ldots]^{15} »$.

Dansl'éditionde 1630, enrevanche, lesmots « etquibusdam aliis 》 sont remplacés par « et Gulielmo Vario » ${ }^{16}$. Faut-il y voir un ajout de Nicolas Rigault et Pierre Dupuy, d'après d'autres manuscrits laissés par de Thou? Ou bien une intervention des éditeurs motivée par le souhait de réparer un oubli? C'est en tout cas ce dernier état du texte que suit la traduction française publiée à Londres en 1734, sur laquelle s'appuie Radouant :

«On avoit agité dès le commencement de l'assemblée des Etats la question du concile de Trente. Le légat en pressoit vivement la publication, et soustenoit que sans la réception pure et simple de ce concile, on ne pourroit maintenir la religion, pour laquelle on combattait depuis si longtemps. La chose ayant été mise en délibération le 9. d'avril, plusieurs objecterent les droits et privileges de l'Eglise gallicane. Ainsi la publication du concile ayant esté jusqu'alors differée, on choisit Jean Le Maitre, que le duc de Mayenne avoit fait president au Parlement, avec Guillaume Du Vair, tous deux éloignés de l'esprit de faction, qui avoient autant de probité que de lumières, pour en examiner les actes, et remarquer ce qu'ils trouveroient estre contraire à la discipline, aux lois et aux usages de ce royaume ${ }^{17}$. »

La convergence de sources distinctes incite donc à confirmer la présence de Du Vair dans la commission chargée d'examiner les décrets du concile. Mais il est difficile de savoir comment les commissaires se sont divisé le travail, et quels furent exactement le rôle et l'influence de Du Vair. Le travail des députés fut de toute façon collectif, dans l'élaboration comme dans la présentation des

14. Voir le Supplément au journal du règne de Henri IV tiré d'un manuscrit du temps et imprimé pour la première fois en 1736, dans les Mémoires-Journaux de Pierre de l'Estoile, éd. Gustave Brunet et al., Paris, Société des Bibliophiles, 1878, t. V, p. 360-361.

15. Jacques-Auguste de Thou, Historiarum sui temporis libri CXXXVIII, Genève, P. de la Rovière, 1620, 1. CV, t. IV, p. 320.

16. Editio secunda, Genève, P. de la Rovière, 1630, t. V, p. 319.

17. Histoire universelle de J. A. de Thou, Londres, 1734, t. XI, p. 711. 
résultats. Ils se sont d'ailleurs appuyés sur des travaux antérieurs, et en particulier sur un mémoire rédigé par l'avocat du roi Cappel ${ }^{18}$. Les résultats de cette enquête sont connus grâce à une plaquette publiée dans les années 1593-1594, et intitulée : Extrait du registre de l'assemblée tenue à Paris sous le nom d'Estats en l'an 1593. Du vendredi neufiesme jour d'avril $1593^{19}$. Le texte se présente comme les extraits « d'aucuns articles du Concile de Trente, qui semblent estre contre et au prejudice de la justice royale et liberté de l'Eglise catholique ${ }^{20} \gg$. Cette plaquette reprend les articles du Concile incriminés, avec les références des sessions en marge, et ajoute ensuite les références des édits royaux ou des lois et coutumes qui seraient remis en cause par ces articles.

Un certain nombre de libertés traditionnelles sont ainsi réaffirmées : - les prérogatives de la justice royale sur certains crimes et délits civils ou religieux, pour le jugement comme pour l'application des peines; - le droit de faire juger les ecclésiastiques français en France, soit par la justice religieuse, soit par la justice royale; la procédure d'appel comme d'abus, en cas d'excommunication notamment; - le privilège royal de gestion du temporel de l'Église de France («le roy ne peut estre privé de son temporel ou partie d'iceluy, pour le regard duquel il ne recognoist aucun superieur quel qu'il soit »).

Le rapport de la commission aboutit très logiquement à une nouvelle suspension des décrets en France. Une forme d'alliance entre les Politiques et certains Ligueurs comme d'Orléans s'était organisée autour de la défense des libertés de l'Église gallicane. Voyant le risque de division du parti ligueur sur cette question, le légat décida de suspendre sa demande pour l'immédiat, en donnant la priorité à la question de la succession et de l'élection d'un roi catholique. Les gallicans l'avaient emporté.

Du Vair avait participé en bonne place à cette bataille. Les événements qui suivirent lui donnèrent un rôle encore plus apparent. Il s'agissait de plaider pour la légitimité d'Henri IV, qui s'était attiré la méfiance du pape et des Ligueurs en raison de sa confession. Du Vair épouse le combat des « Politiques », qui vise à la fois à affirmer

18. Cité dans les Procès verbaux, op. cit., p. 80.

19. BnF, Rés. B 7758.

20. Extraits du registre..., op. cit., p. 3. 
la précellence du principe politique sur le principe religieux, et à réclamer le droit pour l'Église de France de recevoir la conversion du roi sans dépendre de l'assentiment de Rome.

\section{LA DÉFENSE DE LA LÉGITIMITÉ D’HENRI IV}

$\mathrm{Du}$ Vair, qui soutint d'abord la réaction mayenniste contre les Seize en 1591, se rallia à Henri IV à partir de 1592, et il écrivit plusieurs libelles pour défendre sa légitimité. Le premier s'intitule l'Exhortation à la paix ${ }^{21}$; il est diffusé fin 1592. Du Vair s'y aligne sur la position des «semonneux» qui, comme Pierre Pithou et Mathieu Lavergne, veulent « semondre » Henri IV de se convertir. Ils reconnaissent sa légitimité mais exigent sa conversion avant de lui faire allégeance ${ }^{22}$. Du Vair espère lui aussi que la conversion du roi est proche, et il travaille à convaincre les sceptiques qu'elle résoudra toutes les difficultés.

Le second texte de Du Vair en faveur d'Henri IV est la Suasion pour l'arrêt sur la manutention de la loi salique ${ }^{23}$. Il s'agit au départ d'un discours prononcé au Parlement pour défendre l'arrêt du président Le Maître (28 juin 1593); Du Vair en a publié plus tard (en 1606) une version écrite, sans doute largement remaniée. Il y réaffirme la loi fondamentale de la succession des rois de France, qui permettait en l'occurrence d'écarter l'hypothèse d'une reine espagnole. Cela revenait à affirmer la légitimité d'Henri IV avant toute considération religieuse. La succession royale ne devait pas dépendre d'une élection (quelles qu'en fussent les modalités) mais être dictée par les traditions antiques du royaume de France, qui avaient fixé des règles héréditaires précises. Du Vair souscrit dans ce texte au primat du politique sur le religieux, principe caractéristique des positions des « Politiques».

Du Vair écrit un troisième libelle défendant la légitimité du roi, qui nous intéresse particulièrement ici puisqu'il fait la part belle au

21. Exhortation à la paix à ceux de la Ligue, repris dans G. Du Vair, Actions et Traictez oratoires, éd. R. Radouant, Paris, STFM, 1911, p. 63-109.

22. Voir Michael Wolfe, The Conversion of Henri IV, Cambridge (Mass.), Harvard University Press, 1993, p. 112.

23. Suasion de l'arrest donné au parlement pour la manutention de la loi salique, repris dans G. Du Vair, Actions et Traictez oratoires, éd. R. Radouant, op. cit., p. 110-144. 
gallicanisme : la Response d'un bourgeois de Paris à la Lettre de Mgr le Légat du 27 janvier 159424. Ce libelle anonyme conteste le refus du pape Clément VIII de reconnaître l'abjuration d'Henri IV. Il répond à un libelle publié en latin et en français par le cardinal de Plaisance pour défendre la décision du Pape ${ }^{25}$. Si Du Vair choisit l'anonymat pour s'adresser aux catholiques parisiens qui se méfient encore de la conversion du roi, c'est pour ne pas déclarer d'emblée son appartenance au parti royaliste. Il cherche à rassurer les indécis, et fait alterner les concessions aux ligueurs et les arguments en faveur d'un ralliement au roi nouvellement converti. On ne saurait donc chercher dans ce texte la doctrine profonde de Du Vair : il s'agit plutôt d'un témoignage de son action politique en faveur du roi, telle qu'elle s'est exprimée dans une situation d'énonciation bien spécifique.

Dans ce texte, Du Vair réaffirme l'antériorité et la supériorité juridique des lois du royaume de France en matière de succession royale, indépendantes de toute prise en compte du facteur religieux. Il refuse de surcroît l'ingérence étrangère (du pape comme du roi d'Espagne) dans les affaires intérieures françaises. Il lie enfin la défense de l'État et celle de la religion catholique : le royaume de France ne peut être florissant que si la paix revient; celle-ci implique la reconnaissance au moins temporaire de la religion réformée (tolérance civile). Défendre l'État et son autonomie, c'est défendre la paix et l'ordre, et donc la religion. Défendre la guerre (sous le prétexte fallacieux de défendre la religion) c'est nuire à la fois à la religion et à l'État.

Par les thèmes qu'il développe, Du Vair se rapproche clairement des thèses gallicanes défendues par certains évêques depuis 1591 , et en particulier par Renaud de Beaune, archevêque de Bourges ${ }^{26}$.

24. Response d'un bourgeois de Paris à la lettre de Mgr le Légat du vingtseptiesme janvier 1594, repris dans G. Du Vair, Actions et Traictez oratoires, éd. R. Radouant, op. cit., p. 145-188.

25. La Lettre de Mgr l'illustrissime et reverendissime Cardinal de Plaisance, Legat de N. S. Pere et du S. Siege apostolique au royaume de France à tous les catholiques du mesme royaume par lesquelles est déclarée l'intention de Sa Sainteté touchant ce qui s'est nagueres passé à Rome, Paris, Rollin Thierry, 1594.

26. Voir sur ce personnage la notice de Jacqueline Boucher dans Arlette Jouanna et al., Histoire et dictionnaire des guerres de Religion, Paris, Robert Laffont, 1998, p. 706-707. Du Vair était déjà proche de lui en 1588 : il rédigea la version écrite de l'oraison funèbre de Marie Stuart, un texte favorable aux Guises, prononcé par Beaune (voir René Radouant, op. cit., chapitre VII). Beaune tout 
Grand aumônier du roi, il supervise au début du règne la distribution des bénéfices et les nominations ecclésiastiques. Il instruit le roi et lors de la cérémonie d'abjuration, à Saint Denis, le 25 juillet 1593, il posera les questions rituelles auxquelles le roi devra répondre.

À la conférence de Suresnes d'avril 1593, face au ligueur Pierre d'Espinac, archevêque de Lyon, l'archevêque de Bourges a défendu à nouveau la légitimité du roi, dont il a laissé entrevoir la conversion prochaine ${ }^{27}$. Ligueurs et Politiques s'affrontent en fait sur la question des limites du pouvoir du pape en France et sur celle des éventuelles prérogatives de la conscience royale sur les actes pontificaux. Selon Renaud de Beaune et les gallicans, la royauté d'Henri est un droit premier qui ne dépend ni de sa religion, ni des décisions de l'Église. Les concessions du roi en matière religieuse sont accordées par sa libre décision. La couronne est héréditaire, et parler d'élection ou de suffrages est inacceptable. Les libertés gallicanes doivent être préservées et ne permettent pas aux papes d'intervenir en ce qui touche à la succession royale. La loi salique, en désignant clairement le successeur légitime, permet ainsi d'éviter les déchirements des guerres de succession. Le roi ne tient sa couronne que de sa naissance et de Dieu.

Pour d'Espinac, au contraire, il est légitime de choisir un roi qui présente des garanties en matière religieuse. C'est dans cet esprit que le 10 mai 1593 Mayenne portera devant les États généraux la question de l'élection du roi. Pour les Ligueurs, il appartient au pape de décider si la conversion du roi est valable. L'absolution du roi est un préalable obligatoire à tout ralliement.

$\mathrm{Du}$ Vair participe à sa façon au combat des gallicans en faveur du roi nouvellement converti. Dans la Response d'un bourgeois de Paris, il développe un argumentaire soigneusement pesé. Il n'hésite pas à attaquer la politique du pape : celui-ci aurait dû recevoir les émissaires du roi de France, venus implorer son pardon et demander que sa conversion soit reconnue par le Saint-Siège. En rejetant le roi de France, il a pris le risque d'un schisme entre l'Église de France et

comme Du Vair étaient par ailleurs des amis de Christophe de Thou, mort en 1582.

27. Voir Auguste Poirson, Histoire du règne de Henri IV, Paris, Louis Colas, 1856, t. I, p. 177; Arlette Jouanna, La France du XVI siècle, Paris, PUF, 1996, p. 612 et suiv. 
l'Église universelle ${ }^{28}$. Il rappelle au légat (et par son intermédiaire au pape lui-même) le bon usage de l'excommunication, qui ne doit pas viser à empêcher le retour en grâce du pêcheur repenti ${ }^{29}$. Il rappelle le pape à son devoir de mansuétude.

À l'attention des Ligueurs, il souligne qu'à présent que le roi s'est converti, la guerre est devenue sans objet. Il rappelle aussi qu' « en l'extrême nécessité, il est loisible de faire paix avec [un Prince autre que Catholique], et le reconnaître pour seigneur temporel $^{30} »$. La guerre est juste si elle sert une juste cause, mais à condition de ne pas mettre en danger le royaume lui-même. Celui-ci étant désormais à feu et à sang, la paix est devenue urgente. Et même si l'on admettait, avec les Ligueurs, que l'hérésie « rend incapable celuy qui en est infecté de la succession du Royaume par la loi fondamentale d'iceluy », cet obstacle au ralliement est tombé puisque le roi « ayant abjuré [l'hérésie] et fait profession de la foy catholique, $[\ldots]$ a levé l'obstacle qui le rendait incapable du Royaume $»^{31}$. Du Vair rejoint une position gallicane lorsqu'il distingue cet obstacle interne à la conscience du roi de France de l'obstacle intrusif interposé par le pape lorsqu'il refuse de lever l'excommunication. Cette décision, illégitime et abusive, ne doit pas avoir d'incidence légitime sur les affaires intérieures françaises : « Quant à l'excommunication, c'est la note de l'Eglise qui [prive le fidèle] de la communion d'icelle et des graces spirituelles, mais qui en France n'a aucun trait ni consequence au temporel, ni moins à la couronne, qui depend nuement et immediatement de Dieu $»^{32}$.

Du Vair ajoute que cette indépendance temporelle du royaume de France bénéficie, pour finir, à la chrétienté tout entière, qui a besoin que ce royaume - son « bras droit » - reste solide : « Si les interdits et excommunications eussent eu quelque puissance à priver noz rois du Royaume, il y a longtemps que la chrétienté eut ploré la ruine de cest Estat, et en consequence d'icelle eust peut-estre veu la sienne ». Ainsi les royalistes combattent-ils Rome dans l'intérêt

28. Response d'un bourgeois de Paris, dans les Actions et Traictez oratoires, op. cit., p. 155.

29. Ibid., p. 156 (il allègue saint Augustin, Contra epistulam Parmeniani, II, 13).

30. Ibid., p. 167.

31. Ibid., p. 168.

32. Ibid. 
même de l'Église et pour ainsi dire « pour elle mesmes contre elle mesmes $»^{33}$. Il conclut en affirmant résolument l'indépendance du spirituel et du temporel : «quand le roy demeureroit excommunié et interdit, cela ne nous empecheroit point de pouvoir faire paix et de le recognoistre $»^{34}$.

Cependant, Du Vair ne va pas jusqu'à souhaiter durcir l'opposition avec Rome (au moins dans ce texte destiné, avant tout, aux Ligueurs susceptibles de se rallier au roi). «Ce seroit chose fort triste et mal omineuse de voir hors de l'Eglise le Prince qui doit commander à un Royaume tres-chrestien $»^{35}$. C'est pourquoi «il faut toujours conserver, autant qu'il seroit possible, l'union de l'Eglise et rendre au saint siege tout l'honneur qui se pourroit ${ }^{36}$. Du Vair évoque enfin la possibilité pour les parlements de France de suspendre les décisions spirituelles émanant de Rome en cas d'abus constaté, un thème classique des revendications gallicanes :

«L'on a toujours tenu que les sacrements de l'Eglise operent effectuellement en ceux qui font ce qu'ils peuvent pour les recevoir, et particulierement en France le refus que l'on a fait à Rome de ce qui est de la juridiction, voires mesmes des graces ordinaires, a toujours esté reputé pour l'effect; et sur l'attestation du refus, les Parlemens de ce royaume ont contraint les Evesques et Juges ordinaires de l'octroyer. Ce sont les libertez de l'Eglise gallicane, qui ont toujours esté inviolables en ce royaume, et sans lesquelles il ne pourroit subsister : avec lesquelles lon a toujours empesché que la discipline Ecclesiastique ne fust alterée et destournée à l'oppression des particuliers ou ruine de l'Estat. ${ }^{37}$ »

Du Vair propose alors d'appliquer le droit gallican des appels comme d'abus à la personne royale elle-même, qui doit être garantie par le Parlement d'une excommunication abusive. Du Vair réfute ensuite que l'on puisse conserver la religion tout en ruinant l'État (ce que tend à produire la guerre civile, si elle se prolonge encore). Les États sont « les principaux et plus excellens ouvrages de Dieu $»^{38}$. L'État est en effet béni par Dieu, parce qu'il garantit la paix :

33. Ibid., p. 169.

34. Ibid., p. 170.

35. Ibid.

36. Ibid., p. 171.

37. Ibid., p. 170-171.

38. Ibid., p. 177. 
« S'il y a rien en ce monde où Dieu se doive complaire, c'est en l'union et assemblée de tant de peuples, réunis par l'obeissance et temperant leurs mouvemens et moderant leurs mœurs par la regle des lois. Et au contraire il n'y a rien qui lui doive tant desplaire que les ruines des Empires et des Royaumes : car elles ne peuvent aucunement arriver, sinon que par la confusion, par les pillages, brigandage, sang, meurtre, violemens, sacrileges, impieté $\gg{ }^{39}$.

Tout l'irénisme des Politiques est ici contenu. Du Vair montre ensuite que la guerre nuit directement à la religion même, par les exactions et le désordre qu'elle favorise, et qu'elle n'affaiblit pas pour autant l'hérésie. Le libelle se termine par une violente attaque contre le cardinal de Plaisance, accusé d'intelligence avec l'ennemi espagnol.

L'analyse de ce texte montre ainsi deux choses : d'une part, Du Vair souscrit pleinement aux thèmes défendus à cette date par les «Politiques »: gallicanisme, loyalisme monarchique, irénisme. D'autre part, comme la Réponse d'un bourgeois de Paris s'adresse aux Ligueurs modérés, que Du Vair espère rallier au roi avant son entrée à Paris, il est clair qu'il pense qu'un certain nombre des thèses gallicanes sont potentiellement recevables par cet auditoire. L'appel aux libertés de l'Église gallicane ou la critique des excès d'ingérence du pape ne sont donc pas l'apanage des « Politiques »: on l'avait déjà constaté à propos de l'examen des décrets du concile de Trente par les États de la Ligue.

Pour mieux contextualiser les positions défendues par Du Vair, il faut les rapprocher des définitions que Pierre Pithou, très proche de Du Vair en ces années, donne des libertés de l'Église gallicane. Elles sont à l'arrière-plan du libelle de Du Vair. Pithou résume ainsi les principes de son gallicanisme :

«Ce que nos peres ont appelé libertez de l'Eglise gallicane et dont ils ont esté si fort jaloux ne sont point passe droits ou privileges exorbitants mais plutot franchises naturelles et ingenuitez ou droits communs [...] esquels nos ancestres se sont tres constament maintenus. [...] Les particularitez de ces libertez pouront sembler infinies, et neanmoins, estant bien considerees, se trouveront dependre de deux maximes fort connexes, que la France a toujours tenues pour certaines. La premiere est, que les papes ne peuvent rien commander ny ordonner, soit en general ou en particulier, de ce qui concerne les choses temporelles ès pays et terres de l'obeissance et souveraineté du Roy tres-chretien et, s'ils y commandent ou statuent quelque chose, les sujets du roi, 
encore qu'ils fussent clercs, ne sont tenus de leur obéir pour ce regard. La seconde, qu'encore que le pape soit recogneu comme suzerain ès choses spirituelles : toutesfois en France la puissance absolue et infinie n'a point de lieu, mais est retenue et bornee par les canons et regles des anciens conciles de l'Eglise receus en ce royaume ${ }^{40}$. »

$\mathrm{Au}$ fond, Du Vair n'a fait qu'appliquer ces principes généraux à une situation polémique particulière. Pithou, de son côté, fit de même : il publia un opuscule en latin dans lequel il défendait lui aussi la légitimité de la conversion du roi : le De justa et canonica absolutione Henrici IIII, christianissimi Franciae et Navarrae regis $^{41}$, qui fut traduit en français sous le titre suivant : Advis de quatre fameuses universités d'Italie sur l'absolution du Roy. Auquel par le tesmoignage des canons et ordonnances des papes, on preuve que les evesques et prelats de France ont peu absoudre Sa Majesté. Traduit de leur latin en françois ${ }^{42}$. Comme celui de Du Vair, ce libelle s'adresse aux Ligueurs qui seraient prêts à se rallier. Pithou cite les ordonnances et les décrets pontificaux qui permettent de légitimer l'absolution de l'excommunication du roi par les évêques français, en raison d'une part des risques qui pèsent journellement sur sa vie (combats, attentats), et d'autre part des obstacles liés aux devoirs de sa charge, qui rendent son voyage à Rome impossible. Il rappelle aussi que le roi a envoyé des ambassades à Rome, avant et après sa conversion, pour protester de sa bonne volonté auprès du pontife.

Les deux amis utilisent ainsi leur culture gallicane dans une conjoncture politique précise, comme une arme juridique opposable aux tentatives d'ingérence extérieure. Dans le cas de Du Vair, on peut ainsi faire un parallèle entre la défense de la loi salique, rempart contre la candidature de l'Infante espagnole au trône de France, et la défense des libertés gallicanes, qui permettent de valider la conversion du roi contre les menées du pape, et de légitimer son accession au trône de France. La crise de la Ligue viendra bientôt à son terme, mais l'engagement gallican de Du Vair trouvera à nouveau à se manifester, dans un contexte tout différent, celui de la paix retrouvée.

40. [Pierre Pithou], Les libertez de l'Eglise gallicane, Paris, Mamert Patisson, 1594 (début).

41. Paris, Mamert Patisson, in off. R. Stephani, 1594, et Paris, Claude de Monstr'œil et Jean Richer, 1595.

42. Lyon, Guichard Jullieron et Thibaud Ancelin, 1594. 


\section{Du Vair au parlement de Provence}

Envoyé en Provence en 1596 pour redresser l'appareil de la justice royale, Du Vair est resté fidèle à sa sensibilité gallicane. Plusieurs signes le manifestent. Sa méfiance envers les jésuites se laisse lire dans des lettres à Villeroy ${ }^{43}$ (qui est, à Paris, son relais auprès de la Cour). Selon lui, les jésuites sont des hommes de lettres charmants et d'agréable compagnie, mais ils cherchent à s'implanter et à détourner toutes sortes de ressources à leur profit. Leur double inféodation, à Rome et à l'Espagne, les rend particulièrement dangereux dans cette province fraîchement ralliée à Henri IV.

Une autre anecdote est parlante elle aussi : selon des propos rapportés dans des ana, Du Vair se serait vanté d'avoir refusé d'obéir à la reine qui lui demandait de protéger un livre ultramontain de Bellarmin, le Tractatus de potestate summi pontificis in temporalibus, adversum Guillelmum Barclaium ${ }^{44}$. Les doctrines ultramontaines de ce livre avaient été condamnées par le Parlement de Paris, le 26 novembre 1610. Du Vair voit dans ce livre une atteinte au pouvoir absolu des rois de France, et s'étonne que la reine puisse en désirer la protection, au risque de sembler s'engager pour le pape et contre le roi. Il décide que le parlement d'Aix appliquera les décisions du Parlement de Paris ${ }^{45}$.

Premier président du parlement d'Aix à partir de 1599, Du Vair se trouve en butte, à de multiples reprises, aux tentatives de l'archevêque d'Aix pour empiéter sur les prérogatives de la justice royale. Il trouve là l'occasion d'exprimer une certaine conception de la suprématie du politique sur le spirituel, qui n'est pas étrangère à son gallicanisme.

L'archevêque d'Aix, Paul Hurault de L'Hospital, sieur de Vallegrand, était connu pour son caractère ombrageux. Il fut par exemple impliqué dans une affaire de duel (pour un libelle diffamatoire) dès 1591 à Tours, lorsqu'il n'était encore que conseiller dans le Parlement en exil ${ }^{46}$. Il était le petit-fils du chancelier, et le p. 613.

43. lettre du 25 janvier 1610, Revue d'Histoire Littéraire de la France, 1900,

44. Rome, 1610.

45. Anecdotes de l'histoire de France, tirées de la bouche de M. le garde des sceaux Du Vair et autres, éd. L. Lalanne, Paris, 1858, p. 306-307.

46. Voir Laurence Augereau, La Vie intellectuelle à Tours pendant la Ligue (1589-1594), thèse de l'Université de Tours, 2003, t. I, p. 337-345. 
frère cadet de Michel, polémiste qui appartenait au mouvement des «Politiques $»^{47}$. Il arriva à Aix le 9 juin 1595; Henri IV l'envoyait remplacer l'évêque ligueur Génébrard ${ }^{48}$.

$\mathrm{Du}$ Vair venait d'un tout autre univers politique : un moment proche de la Ligue, sympathisant des Guises, partisan de Mayenne à ses débuts, il faisait partie des Ligueurs modérés ralliés au bon moment à Henri IV. Il avait tout pour se concilier les élites d'une province longtemps ligueuse. Il arriva à Marseille en 1596, pour animer une chambre de justice extraordinaire. Il fut nommé en 1599 premier président du parlement d'Aix, après la dissolution de la cour de Marseille. Les fonctions de Du Vair dépassaient de beaucoup l'administration de la justice : il était impliqué dans la politique locale, dans la prévention des complots, des invasions étrangères, des révoltes, etc. Il était en contact épistolaire constant avec le roi ${ }^{49}$.

Paul Hurault n'a certainement pas apprécié de se voir supplanté par Du Vair à la tête d'une région où il était pourtant arrivé le premier. Une lettre de Du Vair à de Thou témoigne que son arrivée en Provence est mal perçue dès l'origine par Paul Hurault, qui tente d'empêcher sa venue :

« J'ay esté là huit jours pour prendre congé du roy, et le jour qu'il me le devoit bailler, M. de Vallegrand luy donna je ne sais par quel esprit tant d'appréhension de mon voyage, qu'il le fist derechef mettre en deliberation en son conseil pour le moins entre quatre ou cinq des principaux. ${ }^{50} »$

Il est difficile de reconstituer la chronologie des querelles de préséance qui s'engage ensuite. Paul Hurault avait obtenu des Lettres de conseiller d'honneur qui lui donnaient voix délibérative au Parlement. La Compagnie fit des difficultés pour le recevoir. Après des délais, elle lui signifia qu'en qualité de procureur de la province, il ne pourrait siéger dans les procès où celle-ci serait intéressée. En

47. Sur Paul Hurault, nous renvoyons également aux travaux en cours de Patrice Alex. Sur son frère Michel Hurault, voir Myriam Yardeni, «La Pensée politique et sociale de Michel Hurault », Revue d'Histoire économique et sociale, 1968, p. 381-402.

48. César de Nostredame, Histoire et chronique de Provence, Lyon, S. Rigaud, 1614, p. 906 et 913.

49. Voir Wolfgang Kaiser, "Guillaume Du Vair et la pacification de la Provence », Guillaume Du Vair, parlementaire et écrivain (1556-1621), éd. B. Petey-Girard et A. Tarrête, Genève, Droz, 2005, p. 109-122.

50. Lettre à de Thou, Lettres inédites de G. Du Vair, éd. P. Tamizey de Larroque, op. cit., p. 17. 
représailles, l'archevêque organisa un incident dans la cathédrale : la plus haute stalle se trouva occupée par l'évêque de Sisteron lorsque Du Vair, accompagné par tous les parlementaires, voulut s'y asseoir (31 janvier 1600) ${ }^{51}$.

Ces querelles de protocole révèlent en fait une rivalité institutionnelle entre deux juridictions en partie concurrentes : la justice laïque et la justice religieuse. La chambre Tournelle avait condamné à mort un prêtre d'Arles coupable de viol. Le procureur général avait lancé une procédure d'appel comme d'abus, parce que le prêtre avait seulement été condamné à une peine de prison. L'archevêque refusa de dégrader le prêtre, ce qui était requis en principe pour exécuter la sentence. Finalement la cour d'Aix passa outre et exécuta le criminel. L'archevêque réunit tous les confesseurs et leur ordonna de refuser l'absolution avant Pâques aux magistrats responsables de la sentence, ainsi qu' aux huissiers et au bourreau eux-mêmes. Le Parlement enquêta et fit appel comme d'abus de cette ordonnance du prélat (5 mars 1601) $)^{52}$, lui infligeant une amende de 4000 écus à peine de saisie de son temporel. Il accepta alors de lever l'excommunication des parlementaires.

Les querelles nées autour du De Ecclesiastica et Politica Potestate d'Edmond Richer (Paris, 1611) prennent place dans ce contexte agité $^{53}$. Le livre a été condamné par le concile de Sens, présidé par Du Perron, et il l'est à nouveau par le concile d'Aix, réuni par Paul Hurault de L'Hospital, qui agit sur demande du pape Paul $\mathrm{V}^{54}$. Richer tente vainement de faire appel de ces sentences. Mais le parlement d'Aix fait de la résistance : il refuse de relayer ces condamnations épiscopales, et ne fait pas interdire le livre ${ }^{55}$. Il faut

51. César de Nostredame, Histoire et chronique de Provence, op. cit., p. 1086.

52. Prosper Cabasse, Essais historiques sur le parlement de Provence, Paris, Pihan Delaforest, 1826, I, chap. 23, p. 376.

53. De la puissance ecclesiastique et politique, Paris, 1612. Sur Richer, voir Edmond Puyol, Edmond Richer, Paris, 1876; Edmond Préclin, « Edmond Richer (1559-1631). Sa vie. Son œuvre. Le richérisme », Revue d'Histoire moderne, 1930, p. 241-269 et p. 321-336; Frédéric Gabriel, « Ecclésiologie gallicane et théologie de la primauté : le cas d'Edmond Richer », à paraître.

54. Voir Edmond Préclin, art. cit., p. 259-260.

55. Honoré Bouche, Chrorographie ou description de la Provence, Aix, C. David, 1664, p. 853. Dans sa condamnation, le concile d'Aix signalait toutefois que cette condamnation ne touchait en rien « aux droits du roi et de la couronne de France, et aux immunitez et libertez de l'Eglise gallicane ». 
dire que certains points du livre de Richer trouvaient un écho direct dans les querelles d'Aix : le point 11 sépare la puissance spirituelle et la puissance temporelle, qui seule peut imposer des peines matérielles; le point 13 rappelle que le roi est protecteur de l'Église et peut recevoir des appels comme d'abus; le point 14 affirme que la puissance du pape n'est pas absolue. Le débat sur le livre de Richer en Provence s'intègre ainsi dans la polémique déjà ouverte pour un clair partage des juridictions spirituelles et temporelles entre le parlement d'Aix et l'archevêché.

Une autre querelle de préséance se déroula cette fois au Parlement, en 1614. L'archevêque voulut y entrer avec sa croix épiscopale, malgré les coutumes qui lui interdisaient de porter les insignes de sa dignité lorsqu'il y siégeait. Les huissiers le contraignirent à laisser sa croix dans la chapelle, à côté de la chambre d'audience. L'archevêque présenta alors une requête au Conseil royal.

Un texte témoigne de cet affrontement: la Tres-humble remonstrance $d u$ Parlement contre M. l'archeveque d'Aix. Il n'est pas signé, mais il se trouve annexé aux œuvres de Du Vair à partir de l'édition posthume de 1625, ce qui laisse penser que Du Vair l'a rédigé. Il est vrai que ses exécuteurs testamentaires, autour de Peiresc, n'en souhaitaient pas la publication (pour ne pas remuer de vieilles querelles? $)^{56}$. Le libraire, Sébastien Cramoisy, l'a donc annexé de son propre chef, hors pagination et lorsque le volume était déjà sous presse. Plus tard, l'édition de 1641, préparée par le neveu de Du Vair, Ribier, entérine cette attribution et donne une place dans le sommaire à cette harangue un peu spéciale, dans la section Actions et traictez oratoires ${ }^{57}$.

La remontrance, adressée au roi, ne craint pas de dramatiser les enjeux de l'affrontement : " La procedure [de l'archevêque] estant toleree renverseroit les loix du Royaume, supprimeroit vostre Justice souveraine, esbranleroit les fondements de l'Estat $»^{58}$. Car il s'agit bien d'une rivalité de deux puissances concurrentes, dont

56. Voir René Radouant, Guillaume Du Vair, op. cit., p. 115.

57. Tres-humbles remonstances faites au Roy en Decembre 1614 de la part de la Cour de Parlement de Provence, sur la poursuite faite au Conseil de sa Majesté par Monsieur l'Archevesque d'Aix, contre Monsieur le Procureur general en ladite Cour, pour une pretendue contravention ou entreprise, dans Les CEuvres de Messire Guillaume Du Vair, Paris, Sébastien Cramoisy, 1641, p. 881-888.

58. Ibid., p. 884. 
l'une doit céder à l'autre : « La croix est la marque de sa juridiction metropolitaine, laquelle il ne peut porter, non seulement aux lieux où s'exerce la Justice souveraine de vostre majesté; mais par la disposition mesme du droit canon, il ne la peut porter en presence d'aucun superieur à luy, et ayant juridiction spirituelle au-dessus de lui ». Le procureur général a eu raison de « s'opposer à tout ce qui s'entreprend au prejudice de vostre authorité et des droits de vos juridictions ». Sous prétexte de religion, l'archevêque a voulu saper la puissance royale, « entreprendre sur les droits royaux de juridiction $»^{59}$. Il a auparavant prétendu refuser l'application d'une peine sur un prêtre; il a excommunié des parlementaires, il a fait des assemblées du clergé de la province sans l'autorisation du roi (il s'agit probablement d'une allusion au concile provincial qui a condamné le livre de Richer). Il critique les privilèges de l'Église gallicane et les appels comme d'abus, bref, «poussé d'ambition, pour se faire renommer, et profiter de l'apparence de son zele, il foule aux pieds les lois de l'Etat et les droits de vostre couronne $»^{60}$. Les prélats, « en ce qui concerne la religion, ne doivent rien avoir au-dessus d'eux, aussi en l'ordre, police et gouvernement de vostre Estat ils doivent estre entierement soumis à vos loix et à vos magistrats $\rangle^{61}$.

Le plaidoyer pour le parlement et contre l'archevêque tourne ainsi à la leçon de gallicanisme pour le jeune roi, « à l'entrée de sa majorité $\aleph^{62}$. Il s'agit de prendre prétexte d'une plainte particulière pour développer des points de droit fondamentaux, qui reflètent la conception que se font $\mathrm{Du}$ Vair et ses amis des libertés de l'Église Gallicane, que la tutelle des parlements royaux protége contre les ecclésiastiques ultramontains.

L'affaire connaît un épilogue puisqu'une dernière querelle d'importance, riche elle aussi d'enjeux symboliques, a lieu en 1618, alors que Du Vair est désormais garde des sceaux à Paris. À nouveau l'archevêque prétend ôter la première place aux parlementaires dans son église pour y faire siéger ses assistants. Après plusieurs affrontements, il va jusqu'à faire construire une estrade pour se hausser avec ses assistants au-dessus des membres du parlement. Ceux-ci

59. Ibid., p. 885 .

60. Ibid., p. 887.

61. Ibid.

62. Ibid., p. 881 . 
rendent un arrêt contre l'archevêque, qui fait appel au Conseil du roi. Le parlement dépêche deux conseillers (Glandevès et Peiresc) pour aller plaider sa cause à Paris ${ }^{63}$. Le 3 mai 1623, le Conseil du roi rend un arrêt d'une remarquable équité, limitant la hauteur de l'estrade mais accordant au prélat la présence de deux assistants à sa hauteur...

Parmi ses amis gallicans, Du Vair ne se distingue ni comme un doctrinaire, ni comme un historien, ni même comme un polémiste de premier plan. Cependant il participe pleinement, dans son action politique concrète, au combat contre les ingérences de Rome, en affirmant l'autonomie de la Couronne et en défendant les prérogatives de la justice civile face à la justice ecclésiastique.

Les textes qu'il a réunis dans ses Euvres portent ici et là la marque de ses engagements gallicans. Théoricien de l'effacement des citations au profit d'une parole oratoire fluide et efficace, Du Vair n'accumule pas les autorités au service de son propos ${ }^{64}$. Il préfère les armes du raisonnement et de la clarté. Il se nourrit de l'érudition de ses amis (Pierre Pithou ou Nicolas Le Fèvre) mais il se passe ensuite de ces références savantes pour diffuser plus largement sa pensée. Qu'il s'adresse aux bourgeois de Paris ou à un roi encore adolescent, Du Vair manie un style simple et fort. Conçus pour être largement diffusés, ses textes témoignent aussi, par contrecoup, du large impact populaire des thèmes gallicans, aussi bien dans les milieux parlementaires que dans la culture du jeune Louis XIII et de son entourage.

alexandre.tarrete@paris-sorbonne.fr

63. P. Cabasse, op. cit., II, chap. 1, p. 7 et suiv.

64. Voir Marc Fumaroli, L'Âge de l'éloquence. Rhétorique et "res literaria » de la Renaissance au seuil de l'époque classique, op. cit., p. 503 et suiv. 